KCL-TH-99-36

hep-th/9908129

August 1999

\title{
M-Theory and Hypercharge
}

\author{
N.D. Lambert \\ and \\ P.C. West ${ }^{\star}$ \\ Department of Mathematics \\ King's College, London \\ England \\ WC2R 2LS
}

\begin{abstract}
We discuss the possibility that the electro-weak and strong interactions arise as the low energy effective description of branes in M-theory. As a step towards constructing such a model we show how one can naturally obtain $S U\left(N_{1}\right) \times S U\left(N_{2}\right) \times$ $U(1)$ gauge theories from branes, including matter in the bi-fundamental representation of $S U\left(N_{1}\right) \times S U\left(N_{2}\right)$ which are fractionally charged under $U(1)$.

KEY WORDS: Hypercharge, Brane Dynamics, M-Theory.

PACS: 12.25.Mj, 11.25.Sq, 12.10.Dm, 12.60.Jv.
\end{abstract}

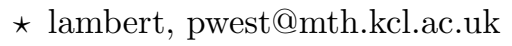




\section{Introduction}

Branes occur as solutions to supergravity theories in ten and eleven dimensions and play a natural role in the dynamics of string theories and the underlying, but elusive, M-theory. From this origin it was somewhat surprising to find that branes had the ability to describe sophisticated properties of four-dimensional quantum field theories that had been studied for many years. These quantum field theories quantum field theories live on the intersection of branes and their dynamics, at low energies, can be computed from the dynamics of the branes. The prototypical example is the spontaneously broken $S U(2) \mathrm{N}=2$ Yang-Mills theory in four dimensions which can be realised on the intersection of NS-fivebranes and D-fourbranes in type IIA string theory. At strong coupling this configuration is lifted to M-theory and appears as a single M-fivebrane wrapped on a Riemann surface [1]. Moreover the complete low energy effective action can be computed from the classical dynamics of the M-fivebrane [2,3] and this agrees precisely with the perturbative $[4,5,6]$ and non-perturbative [7] quantum dynamics of the $\mathrm{N}=2$ Yang-Mills theory.

Given a self intersecting or wrapped M-fivebrane we can compute from the its equations of motion an effective action which describes the low energy behaviour of the configuration. This effective action lives on the self intersection of the M-fivebrane and its degrees of freedom are the moduli corresponding to the M-fivebrane soliton solution that describes the wrapping. The procedure has some similarities to that used to compute the motion of monopoles except now the fields of the underlying theory describe the embedding of the M-fivebrane and the behaviour of its worldvolume gauge field. Since M-theory is the strong coupling description of the type IIA string we can, by shrinking one of the dimensions in the background spacetime in which the M-fivebrane is wrapped, find a corresponding IIA description. In this limit the single M-fivebrane becomes a system of intersecting NS-fivebranes and D-fourbranes. The D-fourbranes have associated with them a non-Abelian gauge theory, the degrees of freedom of which arise as the modes of open strings stretched between the D-fourbranes $[8,9]$. Since these modes also describe the fluctuations of the branes their behaviour provides a description of the the brane configuration in the IIA limit which is the strong coupling limit of that found from the M-fivebrane equations of motion.

An infinite number of the modes of the open strings have masses of the order of the string scale, but there exist a finite number of modes that are either massless or have masses on the scales set by the brane configuration. At energies below the string scale only these latter modes are relevant and one can consider the 
effective action that results from the scattering of the open strings and describes their behaviour. The terms in this action that involve parameters of positive mass dimension will resemble a conventional renormalisable quantum field theory which we refer to as the dual theory.

For the case of $N=2$ Yang-Mills theory mentioned above the complete low energy effective action of the dual quantum field theory including all perturbative and non-perturbative effects agrees with the low energy effective action found from the M-fivebrane equations of motion. However, one would not expect that the higher derivative corrections to agree and indeed it is known that for $N=2$ gauge theories the higher derivative terms of Yang-Mills theory and those from the M-fivebrane dynamics differ [10,3]. While one may not expect the low energy effects of the dual theory and the low energy effective action of the M-fivebrane to agree in all circumstances one would expect a substantial degree of agreement at energies below the scales of the brane configuration. In particular these two theories share the same spectrum of states and gauge symmetry. However if a microscopic description of M-theory were available then we would expect that the brane effective field theory and the dual Yang-Mills effective field theory would agree at scales below the Plank scale.

The M-fivebrane is particularly interesting to study since it has a two-form gauge field in its worldvolume theory which satisfies a non-linear self-duality constraint. There is no known microscopic description available as there is for Dbranes, however the low energy effective dynamics of M-fivebranes are known, although only for the case of a single M-fivebrane corresponding to an Abelian two-form. Now since M-theory is the strong coupling limit of type IIA string theory, the M-fivebrane describes the strong coupling limit of D-fourbranes, including quantum effects. Thus the two-form gauge field contains information about strongly coupled non-Abelian D-fourbrane dynamics, even though the only low energy effective actions that one obtain at present from the M-fivebrane contain only Abelian gauge fields. However these Abelian theories do incorporate some of the non-Abelian aspects of the dual theory, up to the characteristic symmetry breaking scale. For example, the non-linearities in the effective action reflect the scattering of the low energy modes which result from the interactions of the dual theory determined by non-Abelian gauge symmetry, in a manor similar to how the four-Fermi theory accounted for the interactions of the Standard Model. It is also possible to consider M-fivebrane configurations corresponding to confined gauge theories, with no long range gauge group. Although the lack of a microscopic description makes quantitative predictions difficult to obtain. In fact, the degrees of 
freedom that occur in the dual theory that have masses of the order of scales set by the brane configuration also have a description in terms of the dynamics of the M-fivebrane. For example the the solitonic solutions that occur in the underlying non-Abelian theory, in particular monopoles. In Yang-Mills theories these rely on the non-Abelian structure for their existence and do not occur as solutions of the low energy effective action. However their properties can be inferred from the brane dynamics by introducing M-twobranes which intersect the M-fivebrane worldvolume $[1,11,12,13,14]$. In this way the brane configurations mimic the behaviour of the dual Yang-Mills theories.

If String Theory is to play a role in the complete description of physics then it must contain the physics of the Standard Model at low energy. It seems very natural that branes will play a part in the way the Standard Model arises (for some recent work and some reviews see $[15,16,17,18,19,20,21]$ and the references therein). In these scenarios it is usually assumed that the Standard Model itself is the low energy effective action of String Theory, perhaps with some background brane configuration. Moreover it is usually assumed that the minimal supersymmetric extension of the Standard Model, or a grand unified extension of it, is the low energy action of String Theory, which should become valid at some scale near $M_{W}$. In this paper we will take a significantly different approach to deriving low energy effective dynamics of the electro-weak interactions. We will exploit the fact that a single M-fivebrane naturally leads to a low energy effective action with broken supersymmetry, spontaneously broken gauge symmetry whose only unbroken gauge groups are $U(1)$ factor or are confinement.

There is of course very good experimental confirmation that the electro-weak and strong nuclear force is described by the $S U(3) \times S U(2) \times U(1)$ quantum field theory of the Standard Model, at least up to energy scales around its symmetry breaking scale $M_{W}$. As we have explained above, although branes naturally incorporate spontaneously broken gauge symmetry and supersymmetry breaking they do not easily lead to effective theories with unconfined non-Abelian gauge groups. On the other hand, in Nature the only unbroken gauge groups are the confined $S U(3)$ and the $U(1)$ of electromagnetism. Since branes naturally lead to effective theories with these groups, in this paper we explore the possibility that M-fivebrane dynamics can produce a low energy effective theory with the above unbroken gauge groups and that this low energy effective theory will have a dual theory that is the Standard Model, e.g. that this theory has the same spectrum of states as the Standard Model. As we have discussed above this brane derived low energy effective theory will not agree exactly with all the dynamical predictions of 
the Standard Model, but one may hope that they will be sufficiently close to agree with the parts of the Standard Model which have been verified experimentally. If so such a brane configuration would hold out the exciting possibility of new, braney physics beyond the $M_{W}$ scale. We also hope that the work we describe here will be useful for studying other phenomenological models derived from branes.

Although in this paper we will not find such a brane derived low energy effective action we will systematically identify the problems that one encounters and illustrate how some of them may be solved within the context of a toy model. These problems can be thought about in essentially two ways. Firstly we must find a brane configuration with the correct modes (e.g. the dual theory should be the Standard Model). Secondly we must analyse this configuration in M-theory to obtain the brane low energy effective action (e.g. we need to determine the geometry of the M-fivebrane and its resulting zero modes).

One of the most obvious problems is to find a brane configuration which has the gauge group $S U(3) \times S U(2) \times U(1)$. A particular difficulty arises because states in the Standard Model are charged under all three gauge groups, whereas in the D-brane description of branes, states can only be charged under two groups - one for each end point of an open string. A way out of this dilemma is to recall that the brane gauge group that arises from open string theory is $U(N)$, rather than $S U(N)$. In previous studies of gauge theories from branes the additional $U(1)$ factors of $U(N)$ are either trivial and decouple, or they are frozen out of the low energy dynamics. In this paper we will provide explicit examples which rely on compactified spaces and show that this is generically not the case. Furthermore we derive a specific formula for the $U(1)$ charge of state in terms of its representation under $S U\left(N_{1}\right) \times S U\left(N_{2}\right)$. Intriguingly this formula reproduces the correct hypercharges of many fields in the Standard Model, although it also contains fields with the opposite hypercharges.

In section two we discuss how one can find dynamical $U(1)$ gauge groups in the low energy brane theory. We also present a toy brane configuration with fourdimensional $N=2$ supersymmetry whose dual theory is an $S U(3) \times S U(2) \times$ $U(1)$ gauge theory. This model has "quarks" states that transform in the $(\mathbf{3}, \mathbf{2})$ of $S U(3) \times S U(2)$ and have $U(1)$ charge $\pm 1 / 6$. In section three we present a systematic discussion of the problems and issues in constructing a more realistic brane configuration. Section four provides a brief conclusion of our work. 


\section{Hypercharge from Branes}

In this section we wish to construct type IIA brane configurations whose low energy description is a four-dimensional $N=2$ gauge theory where some residual $U(1)$ gauge fields do not decouple. Our strategy is to consider intersecting M-fivebranes in eleven dimensions where $x^{10}$ is compact with period $2 \pi R$. In particular we consider configurations which have four-dimensional Poincare symmetry and preserve $N=2$ supersymmetry, i.e. threebrane solitons on the M-fivebrane. Furthermore we wish to consider M-fivebrane intersections which, when $R \rightarrow 0$ and dimensionally reduced to type IIA string theory in ten dimensions, can be interpreted as configurations of NS-fivebranes with D-fourbranes suspended between them, as first studied in [1]. This then allows us to approach their analysis in two ways. On the one hand we can use perturbative type IIA D-brane physics to calculate the low energy modes and determine the corresponding dual Yang-Mills theory. On the other hand we may solve these models in M-theory where they appear as a single M-fivebrane wrapped on a surface $\Sigma$ and the classical equations of motion provide a good approximation.

It was noted in [1] that one can avoid freezing out all the $U(1)$ factors if the brane configuration is compactified in the $x^{6}$ direction, although only configurations for which the residual $U(1)$ was trivial were considered. In this section we wish to extend this analysis to obtain non-trivial $U(1)$ gauge groups in the dual theory and evaluate their effective action. Therefore we will generalise the so-called Elliptic models in [1], where $x^{6} \cong x^{6}+L$. These are configurations of M-fivebranes which intersect over the four dimensions $x^{\mu}, \mu=0,1,2,3$. Alternatively they can be interpreted as a single M-fivebrane wrapped on a surface $\Sigma$ embedded in $\mathbf{R}^{2} \times \mathbf{T}^{2}$. Demanding that half of the sixteen supersymmetries are preserved requires that $\Sigma$ is a Riemann surface $[22,23]$.

We introduce the complex coordinate $s=x^{6}+i x^{10}$ and the Weierstrass $\wp$ function $\wp(s)$ associated to the torus

$$
s \cong s+\omega_{1}, \quad s \cong s+\omega_{2},
$$

with $\omega_{1}=L$ and $\omega_{2}=2 \pi i R$. As is well-known $\wp$ is a two-to-one map of the flat torus onto $\mathbf{C P}^{1}$ (in other words it is a one-to-one map onto a the twice cut $\mathbf{C P}^{1}$ ) and satisfies the cubic equation

$$
\left(\wp(s)^{\prime}\right)^{2}=4\left(\wp(s)-e_{1}\right)\left(\wp(s)-e_{2}\right)\left(\wp(s)-e_{3}\right),
$$

where $e_{i}=\wp\left(\frac{\omega_{i}}{2}\right)$ for $i=1,2,3$ and $\omega_{3}=\omega_{1}+\omega_{2}$. In this case the limit $R \rightarrow 0$ 
corresponds to going to type IIA string theory on $\mathbf{R}^{9} \times S^{1}$ and the limit $L \rightarrow \infty$ corresponds to uncompactifying this $S^{1}$ factor. Our next step is to introduce a variable $t$ through

$$
t=\wp(s) .
$$

The role of $\wp(s)$ generalises that of $e^{-s / R}$, which maps the cylinder to $\mathbf{C P}^{1}$ in the non-elliptic models of [1].

To analysis our configurations we will consider the limit $L \rightarrow \infty$. In this limit $\wp(s)$ takes the form (see for example [24])

$$
\wp(s)=\frac{1}{R^{2}}\left[\frac{1}{12}+\frac{1}{e^{-s / R}+e^{s / R}-2}\right] .
$$

Note that two of the branch points $e_{1}=\wp\left(\frac{\omega_{1}}{2}\right)$ and $e_{3}=\wp\left(\frac{\omega_{3}}{2}\right)$ have coalesced to $\frac{1}{12 R^{2}}$, whereas the other two branch points are $e_{2}=\wp\left(\frac{\omega_{2}}{2}\right)=-\frac{1}{6 R^{2}}$ and $e_{4}=\infty$. It will be helpful to perform a modular transformation on $t$ so as to map the branch points $e_{2}$ and $e_{4}$ to -1 and +1 respectively and the degenerating branch points $e_{1}$ and $e_{3}$ to $\infty$. Thus we introduce

$$
\tilde{t}=\frac{t+\frac{5}{12 R^{2}}}{t-\frac{1}{12 R^{2}}}
$$

so that, in the limit $L \rightarrow \infty, \tilde{e}_{4}=-\tilde{e}_{2}=1$ and $\tilde{e}_{1}=\tilde{e}_{3}=\infty$.

To describe an embedding of the M-fivebrane we need to specify a function

$$
F(s, z)=0,
$$

where $z=x^{4}+i x^{5}$ is a complex coordinate on the M-fivebrane and $s$ is a complex coordinate transverse to the M-fivebrane. As shown in [22,23], this will preserve four-dimensional $N=2$ supersymmetry for any holomorphic function $F$. A natural form for the embedding of the surface $\Sigma$ into $\mathbf{R}^{2} \times \mathbf{T}^{2}$ is

$$
A_{1}(z) \tilde{t}^{k}+A_{2}(z) \tilde{t}^{k-1}+\ldots+A_{k+1}(z)=0,
$$

where $A_{i}(z)$ are polynomials of degree $N_{i}$ in $z$. 
We may now justify this form for the embedding by considering the limit $L \rightarrow \infty$ where $(2.7)$ reduces to

$$
A_{1}(z)\left(e^{-s / R}+e^{s / R}\right)^{k}+A_{2}(z)\left(e^{-s / R}+e^{s / R}\right)^{k-1}+\ldots+A_{k+1}=0
$$

which is the form of the curve used in [1] for non-elliptic models. Note that $s$ only appears in the combination $s^{-s / R}+e^{s / R}$ so that these brane configurations all have the symmetry $s \leftrightarrow-s$. This is not unexpected from our construction since, when viewed as limit of a configuration which is periodic in $s$, the behaviour as $s \rightarrow \infty$ and $s \rightarrow-\infty$ must be the same. Clearly, for finite $L$, the semi-infinite D-fourbranes going off to the left are identified with those going off to the right.

Let us consider the perturbative type IIA description of this configuration obtained in the $R \rightarrow 0$ limit. After compactification components of the M-fivebrane that are wrapped on $x^{10}$ become D-fourbranes in ten dimensions, whereas the components which are not wrapped on $x^{10}$ become NS-fivebranes. Since $\wp(s)$ is a two-to-one map the surface described by (2.7) consists of a $2 k$-sheeted cover of the $z$ plane with some number of branch cuts on each sheet which is determined by the degrees of the polynomials $A_{i}(z)$. In the type IIA limit each sheet corresponds to an NS-fivebrane while each branch cut appears as a D-fourbrane stretching between two NS-fivebranes. It is helpful to now consider the limit $L \rightarrow \infty$. Due to $A_{1}(z)$, there will be $N_{1}$ D-fourbranes attached to the first NS-fivebrane and $N_{1}$ Dfourbranes attached to the last NS-fivebrane which stretch to $s=-\infty$ and $s=\infty$ respectively. When $L$ is finite these semi-infinite D-fourbranes are identified with each other and become $N_{1}$ finite D-fourbranes stretched between the first and last NS-fivebrane. Note that, and this will be crucial in our analysis, we do not restrict attention to conformal models with the same number of D-fourbranes suspended between all pairs of NS-fivebranes as was studied in [1].

The low energy description of this system is obtained by analysing open strings with end points on the D-fourbranes. The NS-fivebranes are infinitely heavy in the weak coupling limit so that their dynamics are suppressed. Strings that begin and end on the same set of parallel D-fourbranes give a five-dimensional vector multiplet with sixteen supersymmetries and gauge group $U\left(N_{i}\right)$. However, the presence of the NS-fivebranes causes the $x^{6}$ direction of the D-fourbrane to be compactified and projects out half of the supersymmetries and states. This leaves us with a four-dimensional $N=2 U\left(N_{1}\right) \times U\left(N_{2}\right) \times \ldots \times U\left(N_{k}\right)$ gauge theory. We must also consider open strings with one end point on the $i$ th set of D-fourbranes and one end point on the $j$ th set of D-fourbranes with $i \neq j$. These strings give an 
$N=2$ hyper multiplet in four dimensions in the bi-fundamental representation of $U\left(N_{i}\right) \times U\left(N_{j}\right)[1]$.

For example consider the simplest case $k=1$ where

$$
t=\wp(s)=\frac{1}{12 R^{2}}\left(\frac{A_{2} / A_{1}-5}{A_{2} / A_{1}+1}\right) .
$$

In the $L \rightarrow \infty$ limit this is simply

$$
A_{1}(z)\left(e^{-s / R}\right)^{2}+2 A_{2}(z)\left(e^{-s / R}\right)+A_{1}(z)=0,
$$

which corresponds to two NS-fivebranes with $N_{2}$ D-fourbranes suspended between them with $N_{1}$ semi-infinite D-fourbranes coming off to the left and $N_{1}$ semiinfinite D-fourbranes coming off to the right of the NS-fivebranes. For finite $L$ the semi-infinite D-fourbranes are identified and become finite so that we have a four-dimensional $N=2 U\left(N_{1}\right) \times U\left(N_{2}\right)$ gauge theory with a single hypermultiplet in the bi-fundamental representation.

We now need to discuss the low energy dynamics of these configurations obtained from M-theory. In static gauge the bosonic fields of the M-fivebrane worldvolume theory consist of five scalars which correspond to its position in the transverse space and a two-form gauge field whose three-form field strength satisfies a non-linear self-duality constraint. In our case all but two of the scalars are trivial and can be set to zero. The remaining two scalars can be identified with the complex coordinate $s$. To obtain the effective action for the brane configuration we need only expand the equations of motion to second order in field strengths and spacetime derivatives $\partial_{\mu}$. ¿From the constraints of $N=2$ supersymmetry it is sufficient to consider only the scalar zero modes since the rest of the low energy effective action may be determined uniquely from the purely scalar terms. To this end we follow the procedure used in [2], however for more realistic cases with less supersymmetry one must use the analysis of the three-form given in [3]. When the three-form is set to zero the lowest order term in the effective Lagrangian is [2]

$$
\begin{aligned}
\mathcal{L} & =\frac{1}{2} \int d^{2} z \partial_{\mu} s \partial^{\mu} \bar{s}, \\
& =\frac{1}{8} \int d^{2} z \frac{\partial_{\mu} t \partial^{\mu} \bar{t}}{\left|\left(t-e_{1}\right)\left(t-e_{2}\right)\left(t-e_{3}\right)\right|} .
\end{aligned}
$$

To obtain the low energy dynamics of the soliton defined by (2.7), we write the 
$A_{i}(z)$ polynomials in $(2.7)$ as

$$
A_{i}(z)=u_{i, 0} z^{N_{i}}+u_{i, 1} z^{N_{i}-1}+\ldots+u_{i, N_{i}}
$$

and let the moduli $u_{i, a}$ become functions of the four coordinates $x^{\mu}, \mu=0,1,2,3$. The Lagrangian for these scalars can be evaluated as

$$
\mathcal{L}=\frac{1}{8} \int d^{2} z \sum_{i=1}^{k+1} \sum_{a=0}^{N_{i}} \frac{\partial_{\mu} u_{i, a} \partial^{\mu} \bar{u}_{i, a}}{\left|\left(t-e_{1}\right)\left(t-e_{2}\right)\left(t-e_{3}\right)\right|}\left|\frac{\partial t}{\partial u_{i, a}}\right|^{2}
$$

Let us now concentrate on the simplest configuration (2.9) corresponding to a $U\left(N_{1}\right) \times U\left(N_{2}\right)$ gauge theory and write

$$
\begin{aligned}
& A_{1}=u_{0} z^{N_{1}}+u_{1} z^{N_{1}-1}+\ldots+u_{N_{1}}, \\
& A_{2}=v_{0} z^{N_{2}}+v_{1} z^{N_{2}-1}+\ldots+v_{N_{2}} .
\end{aligned}
$$

Here we will be interested in cases where $N_{1}<N_{2}$ and we may therefore rescale $z$ and the curve (2.9) so that $u_{0}=v_{0}=1$. Recall that the zeros of $A_{i}(z)$ represent the positions of the $N_{i}$ parallel D-fourbranes [1]. In particular the sub-leading coefficients $u_{1}$ and $v_{1}$ are the sum of the positions of two sets of parallel D-fourbranes.

Let us now check the convergence of the integral over $z$. For $L<\infty$ the branch points $e_{1}, e_{2}$ and $e_{3}$ are distinct and there are two potential divergences: from the large $z$ limit and from points where $\partial t / \partial u_{i, a}$ diverges. For large $z$ we see that $t \sim 1 / 12 R^{2}$ whereas $\partial t / \partial u_{i, a} \sim z^{-\left(N_{2}-N_{1}+1\right)}$ so that the integrand is well behaved. Next we see that $\partial t / \partial u_{i, a}$ diverges when $A_{2}=-A_{1}$ or when $\partial\left(\frac{A_{2}}{A_{1}}\right) / \partial u_{i}^{a}$ diverges. In the first case $t$ also diverges and this causes the integrand in (2.13) to vanish at these points. The second case can occur when $z \rightarrow \infty$ which we have already considered and potentially at $A_{1}=0$. However when $A_{1}=0$ one finds that $\partial t / \partial u_{i, a}$ is finite. Thus we find that all the moduli $u_{1}, \ldots, u_{N_{1}}$ and $v_{1}, \ldots, v_{N_{2}}$ in $A_{1}(z)$ and $A_{2}(z)$ have finite action for finite $L$. Thus, in contrast to the non-elliptic case, the centre of mass of the D-fourbranes has finite energy. Therefore the $u_{1}$ and $v_{1}$ moduli will appear in the low energy effective action as non-trivial dynamical fields. 
Clearly for any configuration we may shift $z \rightarrow z+z_{0}$ without changing the Lagrangian $\mathcal{L}$. Under this shift the centre of mass moduli are altered according to

$$
u_{1} \rightarrow u_{1}+N_{1} z_{0}, \quad v_{1} \rightarrow v_{1}+N_{2} z_{0} .
$$

It follows that the low energy effective action has a non-trivial dependence on the relative centre of mass between the two sets of D-fourbranes

$$
u_{R}=\frac{u_{1}}{N_{1}}-\frac{v_{1}}{N_{2}},
$$

but depends trivially on the over-all centre of mass

$$
u_{C}=\frac{u_{1}+v_{1}}{N_{1}+N_{2}} .
$$

Therefore although the low energy effective Lagrangian will contain kinetic terms for both $u_{C}$ and $u_{R}, u_{C}$ will be a free field. In addition, due to $N=2$ supersymmetry, the low energy effective action must also have two $U(1)$ gauge fields

$$
\begin{aligned}
A_{\mu}^{R} & =\frac{1}{N_{1}} A_{\mu}^{1}-\frac{1}{N_{2}} A_{\mu}^{2}, \\
A_{\mu}^{C} & =\frac{1}{N_{1}+N_{2}} A_{\mu}^{1}+\frac{1}{N_{1}+N_{2}} A_{\mu}^{2},
\end{aligned}
$$

which are the $N=2$ superpartners of $u_{R}$ and $u_{C}$ at linearised level respectively [3]. However since $u_{C}$ is a free field $A_{\mu}^{C}$ completely decouples from the dynamics and appears trivially in the effective action. On the other hand the effective action will depend non-trivially on $u_{R}$, corresponding to massive states which do carry $A_{\mu}^{R}$ charge that have been integrated out. Therefore our $U\left(N_{1}\right) \times U\left(N_{2}\right)$ brane configuration has a non-trivial $S U\left(N_{1}\right) \times S U\left(N_{2}\right) \times U_{R}(1)$ gauge theory on its worldvolume and we may ignore the fields $u_{C}$ and $A_{\mu}^{C}$.

Let us now examine the charges of the various states under the $U_{1}(1) \times U_{2}(1)$ factor of $U\left(N_{1}\right) \times U\left(N_{2}\right) \cong U_{1}(1) \times U_{2}(1) \times S U\left(N_{1}\right) \times S U\left(N_{2}\right)$. The charge of states from an open string stretched between two distinct D-branes is $(1,-1)$. Here the relative minus sign comes from the orientation of the open string. CPT conjugate states in the $N=2$ multiplet are obtained by considering an open string with the opposite orientation. If an open string begins and ends on the same D-brane then 
it must be uncharged since it is indistinguishable from an open string with the opposite orientation, i.e. it carries $U_{1}(1) \times U_{2}(1)$ charge $(0,0)$. Thus for an open string with one end on a set of $N_{1}$ parallel D-fourbranes and the other on a set of $N_{2}$ parallel D-fourbranes the $U_{C}(1)$ charge always vanishes; $Q_{C}=0$. However the $U_{R}(1)$ charge is

$$
Q_{R}=\frac{1}{N_{1}}-\frac{1}{N_{2}}
$$

From this formula we see that if an open string begins and ends on the same set of D-fourbranes then its $U_{R}(1)$ charge is zero, as one expects since it is in the adjoint representation of $U(N)$. If we consider the conformal cases where $N_{1}=N_{2}$ then the strings stretched between different sets of D-fourbranes are also uncharged under $U_{R}(1)$. In these cases the $U_{R}(1)$ is therefore trivial and can be omitted from the low energy dynamics as in the models of [1]. However in general open strings from one set of D-fourbranes to a different set will yield states with fractional $U_{R}(1)$ charge.

From the point of view of the M-fivebrane each of the low energy $U(1)$ vector gauge fields comes from the period of the Abelian two-form gauge field over a onecycle in $\Sigma$ [3]. However the self-duality constraint of the three-form field strength implies that a one-cycle and its conjugate one-cycle of the Riemann surface give rise to the same low energy $U(1)$. So if $\Sigma$ has genus $g$, we expect $g U(1)$ gauge fields in the low energy effective action. It is easy to see the extra $U(1)$ we have just discussed is associated with the one-cycle corresponding to $x^{6} \cong x^{6}+L$. Thus for a more general embedding with $k>1$ we expect only one additional $U(1)$, corresponding to an $S U\left(N_{1}\right) \times S U\left(N_{2}\right) \times \ldots \times S U\left(N_{k}\right) \times U(1)$ gauge theory, since by compactifying $x^{6}$ we increase the genus of $\Sigma$, and therefore the number of $U(1)$ gauge fields, by one. This was also noted in [1] from a different perspective. To be more explicit we have shown that the one-cycle $x^{6} \rightarrow x^{6}+L$ of the background spacetime coincides with a one-cycle of the embedded surface $\Sigma$. This leads to an additional $U(1)$ gauge field in the low energy effective theory which in the type IIA limit may be identified with the relative centre of mass of the D-fourbranes.

We should comment here on an apparent difficulty: if a brane low energy effective action has $n U(1)$ gauge fields how do we know whether a particular one of these $U(1)$ 's arises from the Cartan subalgebra of $S U(N)$ of whether it arises as a relative $U_{R}(1)$ ? To be more concrete suppose we have a model with two $U(1)$ gauge fields in the low energy effective action. How can we tell if this comes from spontaneously broken $S U(3)$ gauge theory or an $S U(2) \times U(1)$ theory? As one can 
see from above analysis, the appearance of the $U_{R}(1)$ factor is associated with the non-trivial topology of spacetime, i.e. compactness of $x^{6}$. This means that there is no point in the brane moduli space where this non-trivial one-cycle associated to $U_{R}(1)$ degenerates and a non-Abelian symmetry is restored. In contrast for an $S U(3)$ gauge theory there will be points in the brane moduli space, i.e. vacuum expectation values of the scalars, where the gauge symmetry will be enhanced and the corresponding one-cycles in $\Sigma$ are degenerate.

Thus we can construct a toy model for the electro-weak interactions by taking $k=1, N_{1}=2$ and $N_{2}=3$, i.e. we consider the embedding

$$
\left(z^{2}+u_{1} z+u_{2}\right) \tilde{t}+\left(z^{3}+v_{1} z^{2}+v_{2} z+v_{3}\right)=0 .
$$

The corresponding type IIA brane configuration is shown in figure one. The vertical lines denote the NS-fivebranes, the horizontal lines the D-fourbranes and the dashed lines the various possible open strings that can stretch between the Dfourbranes. The non-trivial part of the low energy dynamics consists of an $N=2$ supersymmetric $S U(2) \times S U(3) \times U_{R}(1)$ gauge theory. In addition we have a hyper multiplet of "quarks" in the $(\mathbf{2}, \mathbf{3})$ of $S U(2) \times S U(3)$ with $U_{R}(1)$ charge $\pm 1 / 6$. In fact, since the open strings that stretch between the two sets of parallel $\mathrm{D}$ fourbranes can wrap $n$ times around the compact $x^{6}$ direction, we find an infinite tower of quark "generations" whose masses increase linearly with $n$. Of course this model is far from describing the quarks of the Standard Model. Most notably it is non-chiral, has $N=2$ supersymmetry and the $S U(3)$ factor of the gauge group is spontaneously broken, rather than confined. Nevertheless this model arises naturally and quite simply from brane configurations and the charges of the "quarks" are realistic.

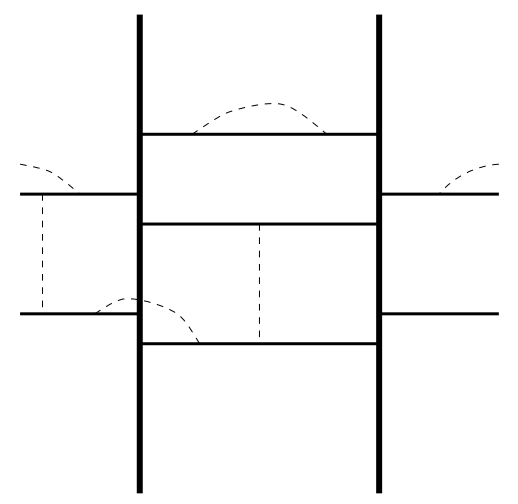

Figure 1 


\section{Towards a Fivebrane Realisation of the Electro-Weak and Strong Interactions}

In this section we wish to explore the possibility that M-theory can describe the electro-weak and strong interactions. More precisely we wish the theory of electroweak and strong interactions to arise from an intersecting M-fivebrane configuration in an eleven-dimensional background spacetime. The effective low energy action can be calculated from M-theory and will live on the worldvolume of the selfintersection of the M-fivebrane. This space is to be identified with the world in which we live and so is taken to be four dimensional Minkowski space $\mathbf{M}^{1,3}$. The remaining two dimensions of the M-fivebrane are wrapped on a two dimensional manifold $\Sigma$ embedded in the background spacetime. This effective action will describe the electro-weak and strong interactions. A necessary condition for this to occur is that the dual gauge theory that arises in this brane configurations as identified in the IIA limit must be a $S U(3) \times S U(2) \times U(1)$ gauge theory which has the same matter content as the Standard Model. In particular the resulting brane configuration that emerges in the IIA limit must have the field gauge group $S U(3) \times S U(2) \times U(1)$ which is spontaneously broken to $U(1)$ and a confining $S U(3)$ and have the field content of the Standard Model. Of course it must therefore have no supersymmetry.

The background spacetime must be a solution of eleven dimensional supergravity of the form $\mathbf{M}^{1,3} \times Q$, where $Q$ is a seven dimensional space. The M-fivebrane dynamics describe the embedding of $\Sigma$ into $Q$. Here we will use the classical equations of motion of the M-fivebrane to determine features of the low energy effective action for this embedding. These are only a good approximation when the manifolds $Q$ and $\Sigma$ are sufficiently large and smooth. Although when considering branes that lead to confining gauge groups this is not always the case, the classical equations of motion along with what is known about the quantum corrections to the M-fivebrane will provide a qualitative description of the low energy dynamics. From the perturbative IIA string theory point of view, for which there is the dual Yang-Mills theory interpretation, an analysis of the classical M-fivebrane dynamics incorporates the strong coupling regime of this theory. The limit to the IIA theory is found by shrinking one of the one-cycles in the background spacetime. Clearly in order to obtain a system of NS-fivebranes and D-fourbranes this cycle must belong to both $\Sigma$ and $Q$. Thus our task is to identify a suitable choice for $\Sigma$ and $Q$ that will lead to a low energy effective action for the M-fivebrane which describes the electro-weak and strong interactions. 
In order to illustrate more clearly the problems that must be solved let us first consider the toy model discussed in the previous section. In this case we consider a background spacetime $Q=\mathbf{T}^{2} \times \mathbf{R}^{2} \times \mathbf{R}^{3}$ where the $\mathbf{R}^{3}$ factor is trivial. The Mfivebrane is wrapped around a Riemann surface $\Sigma$ embedded in $\mathbf{T}^{2} \times \mathbf{R}^{2}$ defined by the embedding (2.20). The self-intersection of the M-fivebrane in spacetime appears, from the perspective of the M-fivebrane, as the presence of threebrane soliton solutions on its worldvolume. Using the classical M-fivebrane dynamics we can compute the low energy effective action for the motion of these threebranes. Indeed the scalar part of this low energy effective action is given by (2.11). The result is a low energy effective action in four-dimensional Minkowski space. More precisely, the degrees of freedom of this low energy effective action arise from moduli in the solution of the M-fivebrane equations of motion. In the threebrane solution only the transverse scalar coordinates of the M-fivebrane are active and changes of the moduli that only affect these coordinates correspond to deformations of the embedded surface on which the M-fivebrane is wrapped. These moduli lead to scalar degrees of freedom in the low energy effective action, namely $u_{R}, u_{2}, v_{2}, v_{3}$. There are also moduli which correspond to a non-vanishing worldvolume two-form gauge field and are associated with large gauge transformations. Such moduli arise as periods of the two-form potential over one-cycles on $\Sigma$ and lead to vector fields in the four-dimensional low energy effective action [3]. For example in the toy model there are four non-trivial one-cycles: two from the set of three parallel Dfourbranes, one from the set of two parallel D-fourbrane and the fourth comes from the $x^{6}$ cycle in spacetime. This leads to an $U(1)^{4}$ vector gauge potential in the low energy effective dynamics. There are four conjugate one-cycles on the Riemann surface, however due to the self-duality constraint on the three-form field strength these do not lead to additional low energy vector fields (this will be discussed in greater detail below). Finally we have Fermionic moduli that lead to spin 1/2 particles in the low energy effective action.

As we explained above, we can consider the limit in which one dimension $x^{10}$ of the background torus is shrunk to zero. In this limit the wrapped M-fivebrane becomes a system of intersecting NS-fivebranes and D-fourbranes in type IIA string theory. The excitations of this brane configuration are described by the open strings stretched between the D-fourbranes. The modes of the open strings can be divided into those that have masses on the string scale, and are therefore rather heavy, as well as a finite number of modes whose masses are much below the string scale. The latter include massless modes for open strings whose ends are attached to the same D-fourbrane or whose ends are fixed to D-fourbranes that touch at one 
or more points, and massive modes for those open strings whose ends are fixed to D-fourbranes that are never touch. The mass of the latter is proportional to the minimum length of the stretched open string and so are fixed by the scales of the brane configuration, rather than the string scale. The lowest energy modes of these open strings correspond to the excitations of the brane configurations. At a first approximations the dynamics of these modes, which are in fact given by open string perturbation theory, can be described by the dual Yang-Mills quantum field theory on the worldvolume of the intersection. Thus using the connection between M-theory and IIA string theory we can identify the dual quantum field theory. In addition the complete low energy effective action is exactly described by the low energy dynamics for the self-intersecting M-fivebranes $[2,3]$.

Although the non-linearity of the low energy effective action can be thought of as arising from integrating out particles such as the non-Abelian gauge fields it is difficult to infer the existence and properties of these particle from the low energy effective action. However, all the particles of the quantum field theory on the brane, as identified in the IIA limit, do occur naturally in the M-theory description. The particles that occur in the IIA limit that have masses on brane scales arise as excitations of the string stretched between different D-fourbranes. In M-theory, since there are only M-twobranes and M-fivebranes, when we lift a IIA configuration the open strings between the D-fourbranes must correspond to M-twobranes which end on the M-fivebrane. These M-twobranes appear on the worldvolume of the M-fivebrane as a self-dual string solitons that carry the twoform gauge field charge [29]. For general configurations, these states occur solitons on the M-fivebrane with non-vanishing worldvolume gauge field corresponding to a self-dual string wrapping $\Sigma[13,14]$. The type of particle one finds depends on how the self-dual string wraps itself around $\Sigma[1,11,12]$. In this way one can find in particular the the charged spin one vector bosons $W^{ \pm}$and the monopoles of the $S U(2) \times S U(3)$ factor. Such M-fivebrane solutions were explicitly constructed in reference $[13,14]$ with in the context of the M-fivebrane wrapped on a Riemann surface embedded in flat spacetime in the case of a single $S U(2)$ gauge group. Although the M-fivebrane equations only described a $U(1)$ gauge theory it was argued in [14] that finite energy solutions corresponding to the non-Abelian vectors and monopoles do exist. The low energy scattering of these particles can also be deduced from the M-fivebrane equations of motion by letting the moduli associated with the solution (including the self-dual string) become dynamical [14].

Thus despite the rather different techniques used to find the two descriptions, one from M-theory and the other from the perturbative IIA string, there is a one- 
to-one correspondence between the spectrum and low energy dynamics of the two theories. Although we have been describing systems with $N=2$, where there are significant constraints, the qualitative features of the dual quantum Yang-Mills theory and M-fivebrane gauge theory should agree for systems with less and even no supersymmetry.

Let us now return to the task in hand, to construct a solution of M-theory that describes the electro-weak and strong interactions. We require a worldvolume threebrane soliton, described by the wrapping of the M-fivebrane on a surface $\Sigma$ embedded in a background spacetime of the form $M^{1,3} \times Q$. Our first restriction on $Q$ arises because gravity is not a low energy mode of the brane and in fact propagates in the full eleven-dimensional spacetime. The simplest way to obtain a $1 / r^{2}$ force law for gravity, rather than a $1 / r^{9}$ law, is compactify all directions $x^{4}, x^{5}, x^{6}, \ldots, x^{10}$ so that gravity is four-dimensional on macroscopic scales. Therefore we assume that $Q$ is a compact manifold. Note that some alternatives where the extra dimensions are non-compact have recently appeared $[25,26,27,28]$.

Since we wish to find a description of the electro-weak and strong nuclear forces the M-fivebrane wrapped on $\Sigma \subset Q$ should break all supersymmetries. There are two ways in which this could happen. Either the background spacetime $Q$ breaks all the supersymmetries of M-theory, or the background preserves some or all of the supersymmetries of M-theory, but the two-cycle $\Sigma$ is not supersymmetric (i.e. is not a calibrated submanifold $[22,30,31,40,32])$. In either case one must ensure that the configuration is stable. In the later case the preservation of some supersymmetry by the background space-time implies that the manifold will possess reduced holonomy. If the background gauge field is zero such manifolds are classified [39] and the only seven-dimensional manifolds which do not have a direct product structure have $G_{2}$ holonomy. The residual supersymmetry is then broken by the wrapping of the M-fivebrane on $\Sigma$.

A systematic study of the possible supersymmetric intersections of a single M-fivebrane was given in reference [40]. If the compact manifold $Q$ has a direct product structure then there are a number of ways of wrapping the M-fivebrane such that supersymmetry is preserved. One example being given in the previous section. However, if $Q$ does not have a direct product structure then there are no background manifolds with two submanifolds $\Sigma$ such that any supersymmetry is preserved by the wrapped M-fivebrane. In particular, $G_{2}$ holonomy manifolds generically possess nonsupersymmetric two-cycles. Even though wrapping a Mfivebrane on such a two-cycle dos not preserve supersymmetry there may exist in 
the background manifold cycles of different dimensions which wrapping the Mtwobranes or other M-fivebranes leads to supersymmetric states. For example, $G_{2}$ holonomy manifolds do possess non-trivial three and four-cycles over which one can wrap M-fivebranes without breaking all the supersymmetry [40]. These would lead to supersymmetric states in spacetime corresponding to topological defects in $\mathbf{M}^{1,3}$. We observe that if we require $Q$ to not possess a direct product structure and the self intersection of the M-fivebrane to be four-dimensional then the resulting low energy effective action will break supersymmetry.

Once one has identified a suitable type IIA brane configuration and its corresponding lift to an embedding $\Sigma \subset Q$ the next step to analyse the low energy effective dynamics and states using the M-fivebrane dynamics. We now describe these two steps in greater detail.

The IIA Limit

As is well known the Standard Model is based on a non-supersymmetric $S U(3) \times S U(2) \times U_{Y}(1)$ Yang-Mills gauge theory which is spontaneously broken to $S U(3) \times U(1)$ and in addition the $S U(3)$ factor is confined. The matter content consists of three generations, which, from a group theoretic point of view are identical but whose masses vary dramatically. Here we shall concentrate on the lightest generation which has the following the matter content

$$
S U(2) \quad S U(3) \quad Y
$$

$\begin{array}{cccc}q_{L} & \mathbf{2} & \mathbf{3} & 1 / 6 \\ e_{L} & \mathbf{2} & \mathbf{1} & -1 / 2 \\ u_{R} & \mathbf{1} & \mathbf{3} & 2 / 3 \\ d_{R} & \mathbf{1} & \mathbf{3} & -1 / 3 \\ e_{R} & \mathbf{1} & \mathbf{1} & -1\end{array}$

where $L / R$ denotes the left/right-handed Weyl component of the spinor field.

As we have discussed above, it is rather straightforward to find brane configurations that lead to the gauge groups $S U\left(N_{1}\right) \times S U\left(N_{2}\right)$; one simply takes $N_{1}$ and $N_{2}$ parallel D branes which are suspended between NS-fivebranes. The additional $U(1)$ factor which one might naively expect to be present is frozen out if one demands that the low energy effective action is finite [1]. One way to avoid such an infinity and so find a $U(1)$ factor is to compactify directions in which the 
D-fourbranes lie in. This occured in the so called elliptic models of [1]. Unfortunately in these models considered there the gauge group was $S U(N)^{k} \times U(1)$ and the $U(1)$ gauge field decoupled from the rest of the theory.

In the previous section we showed that one could compactify one of the directions and find a theory with the gauge group $S U(3) \times S U(2) \times U(1)$ and the hypermultiplets are charged under the $U(1)$ factor. We recall that the existence of an additional $U(1)$ relies on the presence of a one-cycle of $\Sigma$ that coincides with a one-cycle in the background space $Q$. Although this model possess $N=2$ supersymmetry and so is unrealistic it clearly illustrates that one can find the correct gauge group. Let us consider its matter content and postpone the problem that these states form a non-chiral $N=2$ hyper multiplet. The toy model possessed a "quark" that was in the same group representation as $q_{L}$, including the correct $U(1)$ charge (it also possessed a "quark" with the opposite $U(1)$ charge). In addition the toy model had a generation structure, although these states had a linearly increasing mass as a function of their wrapping number $n$, whereas in the Standard Model the mass of the successive generations increases much more rapidly.

The toy model can be made more realistic in a number of ways. To gain a confining $S U(3)$ we can follow $[33,34,35]$ and rotate one of the NS-fivebranes so that it lies in the $x^{7}, x^{8}$ plane rather than in the $x^{4}, x^{5}$ plane. From the geometrical point of view this corresponds to embedding $\Sigma$ non-trivially in six of the dimensions of $Q$. In these configurations there the distance between the NS-fivebranes which connect the three parallel D-fourbranes are no longer constant and therefore the three parallel D-fourbranes will lie along the shortest distance between them. In this case the M-fivebrane worldvolume is no longer a smooth manifold but has a singularity where the D-fourbrane sit on top of each other. The M-fivebrane equations of motion are no longer valid and in addition the system can no longer be described by a single M-fivebrane. The D-fourbranes no longer have any scalar moduli and lead to a confining $S U(3)[34,35]$. Indeed if $Q$ is a curved manifold then one might expect there to be no scalar moduli for a particular embedding since in general the minimal sized cycles and distances will be unique, i.e. there will be no flat directions in the M-fivebrane moduli space. To remove the massless scalar from the broken $S U(2)$ sector of the theory one may suppose that $Q$ is curved so that there are two paths which are (locally) the shortest distance between the NSfivebranes. This would then remove the massless scalar mode that represents the fluctuations of the D-fourbranes but still lead to a spontaneously broken $S U(2)$.

We can also gain a more realistic matter content by introducing additional sets 
of D-fourbranes into the toy model. In particular we can add a single D-fourbrane stretched between additional NS-fivebranes to our configuration in a manner similar to figure two. Although the configuration in figure two is not of the form described in the previous section, it might still be possible to find other embeddings which allow it to be compactified. From the discussion in the last section this would not lead to additional $S U(N)$ or $U(1)$ factors of the gauge group. However open strings stretched from the additional D-fourbrane to the sets of two and three parallel Dfourbranes we would obtain states in the $(\mathbf{1}, \mathbf{2})$ and $(\mathbf{3}, \mathbf{1})$ of $S U(3) \times S U(2)$. Their representation would be the same as for $e_{L}$ and $u_{R}$ respectively, again obeying the $U(1)$ charge formula (2.19) derived in the previous section (up to a sign). On the other hand it is not clear how to obtain states corresponding to $d_{R}$ and $e_{R}$. In this case the formula (2.19) does not appear to work for these states, although it only differs by an additive factor of one.

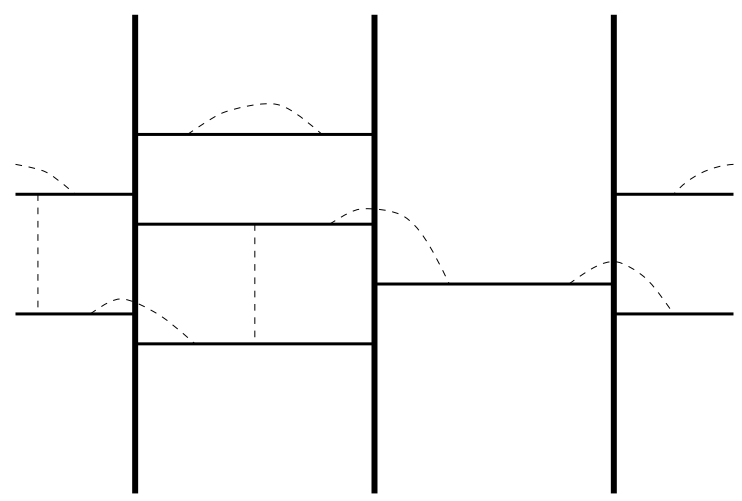

Figure 2

Another key problem with the toy model is that it is non-chiral. The open strings that are used to study a D-brane configuration naturally come with sixteen supersymmetries and produce four-dimensional $N=4$ supermultiplets. By placing NS-fivebranes into the configuration the number of preserved supersymmetries is decreased and the size of the supermultiplet is reduced accordingly [1]. In particular, within the Green-Schwarz formalism, this leads to a mechanism where as supersymmetries are projected out and the multiplet is reduced [36]. In some cases, when all of the supersymmetries are projected out, only the highest spin component of the open string multiplet survives [36]. Thus we might expect that only the spin 1 and spin 1/2 states of the vector and hyper multiplets respectively survive in many non-supersymmetric brane configuration. This is just as is required to 
make contact with the fields of the Standard Model, i.e. vector fields with chiral matter.

The low energy effective action

Let us now consider the requirements that the M-fivebrane wrapping on the two-cycle must possess in order to describe the required low energy effective theory.

One of the remarkable features of the Standard Model is that there are no explicit mass terms in the theory as a consequence of the different multiplet structures of the Weyl left-handed and right-handed Fermions, the gauge symmetry for the vector bosons and the doublet structure of the Higgs. After the symmetry breaking all particles get a mass through the Higgs mechanism except for the photon, gluons and neutrinos. Particles which get their mass in the symmetry breaking must be regarded as associated with the symmetry breaking scale and will not appear as dynamical moduli in the low energy theory. Hence we should only find moduli in the M-fivebrane embedding solution that correspond to the photon, gluons and the neutrino. However as we mentioned above the gluons, due to confinement, are not described by the classical low energy effective action of the M-fivebrane. Instead one needs to know the microscopic theory underlying the M-fivebrane dynamics describe them. All the other particles in the Standard Model should arise as more complicated M-fivebrane solutions consisting of a self-dual string wrapping around the two-cycle $\Sigma$. As we have discussed above these particles arise as modes of the stretched open strings between the D-fourbranes in the type IIA limit.

It is instructive to examine the M-fivebrane equations that must be solved. We use the covariant equations of motion found in [37] which were derived from the superembedding formalism applied to the M-fivebrane [38]. Neglecting the background three-form gauge field the scalars of the obey the equation [37]

$$
G^{m n} \nabla_{m}\left(\partial_{n} X \underline{p}\right)=0
$$

where the covariant derivative $\nabla_{m}$ is a connection with respect to worldvolume and background indices. Here and below underlined indices run over the eleven-dimensional background space $\underline{m}, \underline{n}, \underline{p}=0,1,2, \ldots, 10$, whereas $m, n, p, \ldots=$ $0,1,2, \ldots, 5$ are worldvolume coordinates. To be precise

$$
\nabla_{m} V_{\bar{n}}^{\underline{p}}=\partial_{m} V_{\bar{n}}^{\underline{p}}-\Gamma_{m n}^{q} V_{\bar{q}}^{\underline{p}}+\partial_{m} X^{r} \Gamma_{\underline{\underline{r}} \underline{p}} V_{\underline{n}}^{\underline{s}}
$$

where the connections are the standard Christoffel connections coefficients with respect to the induced metric $g_{m n}$ on the worldvolume and background metric 
$\gamma_{\underline{m n}}$ respectively (note that the last term was neglected in [37]). We also need to introduce tangent indices for the M-fivebrane worldvolume $a, b, c, \ldots$ and the vielbein $g_{m n}=e_{m}^{a} e_{n}^{b} \eta_{a b}$. The tensor $G^{m n}$ is defined as

$$
\begin{aligned}
G^{m n} & =\left(m^{2}\right)^{a b} e_{a}^{m} e_{b}^{n}, \\
m_{a}^{b} & =\delta_{a}^{b}-2 h_{a c d} h^{b c d} .
\end{aligned}
$$

Here $h_{a b c}$ is related to the three-form field strength and will be defined below.

The actual embedding is given by a field configuration in which the worldvolume gauge field $B_{m n}$ vanishes, the flat coordinates $X^{\mu}$ of the self-intersection of the M-fivebrane are taken to belong to four-dimensional Minkowski space; $\partial_{\mu} X \underline{p}=0, \mu=0,1,2,3$. Equation (3.1) then determines the behaviour of the scalar coordinates on the two remaining coordinates $x^{4}, x^{5}$ of the M-fivebrane and specify how it wraps around the two-cycle $\Sigma$ of the background space $Q$.

The scalar moduli of the embedding solution become dynamical when they are allowed to depend on the flat worldvolume which has coordinates $x^{\mu}$ and their equation of motion can be derived from equation (3.1) by dimensionally reducing the equations of motion over $x^{4}$ and $x^{5}$. The behaviour of these moduli can be studied in the absence of worldvolume gauge fields. In this case the equation minimises the volume of $\Sigma$. The smallest volume is likely to be given by the Mfivebrane wrapping tightly to any fixed background cycles. In such a case one will find no scalar moduli as any deviation will lead to a solution with an increased energy. This is a welcome feature as we should find no scalar fields in our low energy effective action.

It is instructive to realise the difference with the toy model studied in section two where scalar moduli were present. In this case the background space had certain flat directions, partly because there was no fixed two-cycle in $Q$ around which the M-fivebrane wrapped. The equations of motion were satisfied by any Riemann surface $\Sigma$ and thus there were corresponding scalar moduli parameterising the moduli space of Riemann surfaces with a fixed genus. If $Q$ had a more complicated topology and geometry then one would not expect to find moduli spaces of solutions with the same energy, but rather a fixed surface $\Sigma$.

Let us now examine how gauge fields can arise in the effective action. The worldvolume three-form field strength $H=d B$ of the M-fivebrane obeys a nonlinear self-duality constraint. This is most easily described by first considering a 
linearly self-dual field $h$

$$
h_{a b c}=\frac{1}{3 !} \epsilon_{a b c d e f} h^{\text {def }} .
$$

Next we construct the field strength $H_{a b c}$ as

$$
H_{a b c}=\left(m^{-1}\right)_{a}^{d} h_{d b c}
$$

A final step is to impose that $H$ is closed, so that we may identify $H=d B$. As a consequence of this non-linear self-duality constraint the closure of $H$ is in fact equivalent to the equation of motion [37]

$$
G^{m n} \nabla_{m} H_{n p q}=0
$$

In general it is rather complicated to solve this system of equations, but to discover the presence of vector fields in the low energy effective action it is sufficient to work to linearised order in $H$ and to zeroth order in derivatives of the scalar with respect to the coordinates $x^{\mu}$. In this case we need only solve $H=\star H$ and $d H=0$. We may write $H$ in the form

$$
H=\mathcal{F} \wedge \omega+k \wedge \Omega+L
$$

where $\omega$ and $\Omega$ are one-forms and two-forms on $\Sigma$ respectively and $k, \mathcal{F}$ and $L$ are one, two and three-forms on $\mathbf{M}^{1,3}$ respectively. To the approximation to which we are working, the equation $d H=0$ implies that

$$
d_{2} \omega=0, \quad d_{4} \mathcal{F}=0, \quad d_{4} k=0, \quad d_{4} L=0,
$$

where $d_{2}$ and $d_{4}$ are the exterior derivatives on $\Sigma$ and $\mathbf{M}^{1,3}$ respectively. Low energy modes arising from $k$ and $L$ contribute additional four-dimensional scalars and thus we must also ensure that these do not occur.

The self-duality of $H$ then implies that

$$
H_{\mu \nu i}=\frac{1}{2} \operatorname{det}(e) \epsilon_{\mu \nu \rho \kappa} \epsilon_{i j} g^{j k} H_{\rho \kappa k j},
$$

where $i, j=4,5$. We may decompose $\omega$ into the self-dual one-forms $\omega_{A}^{ \pm}$which 
satisfy $\omega_{A i}^{ \pm}= \pm i \operatorname{dete} \epsilon_{i j} g^{j k} \omega_{A k}^{ \pm}, A=1,2,3, \ldots, g$. Writing

$$
\mathcal{F} \wedge \omega=\sum_{A=1}^{g}\left(\mathcal{F}_{A}^{+} \omega_{A}^{+}+\mathcal{F}_{A}^{-} \omega_{A}^{-}\right)
$$

we find that the above self duality condition becomes

$$
\mathcal{F}_{A \mu \nu}^{ \pm}= \pm \frac{i}{2} \epsilon_{\mu \nu \rho \kappa} \mathcal{F}_{A}^{ \pm \rho \kappa} .
$$

Defining $\mathcal{F}_{A}^{ \pm}=F_{A} \pm i \star F_{A}$ we conclude that $F_{A}$ is real and satisfies $d F_{A}=0$ and $d \star F_{A}=0$. As such it represents a $U(1)$ gauge field in the low energy effective action. Hence we may conclude that the for every pair of conjugate two-cycles in $\Sigma$, we find a $U(1)$ gauge field in the effective action. For our purposes we require only one gauge field hence only one pair of one-cycles. We recall from section two that the existence of a $U(1)$ factor required a one-cycle in $\Sigma$ that was also a one-cycle in the background spacetime $Q$.

Let us now turn our attention to the worldvolume Fermions $\Theta^{\underline{\gamma}}, \underline{\gamma}=$ $1,2,3, \ldots, 32$. Without gauge fixing these obey the equation [37]

$$
g^{m n} \partial_{n} \Theta \underline{\underline{\gamma}}(1-\Gamma)_{\underline{\underline{\beta}}}^{\underline{\beta}}\left(\Gamma_{m}\right)_{\underline{\alpha} \underline{\beta}}=0 .
$$

where $E_{\underline{\underline{a}}}^{\underline{\underline{a}}}$ is the eleven-dimensional vielbein, $\Gamma_{m}=\partial_{m} X \underline{\underline{n}} E_{\underline{\underline{n}}}^{\underline{a}} \Gamma_{\underline{a}}$ and

$$
\Gamma=\frac{1}{6 ! \sqrt{-g}} \epsilon^{m n p q r s} \Gamma_{m n p q r s}+\frac{1}{3} h^{m n p} \Gamma_{m n p} .
$$

Note that this equation involves a Dirac operator that is quite different to that usually found for Fermions propagating in a curved background. As such the usual discussions of zero modes which are for example found in Kaluza-Klein theories will not apply in an obvious way. The Fermionic moduli are the zero modes of (3.12) in the presence of the threebrane solution. The Fermions that appear in the low energy effective action are found by letting these zero modes depend on $X^{\mu}$ and their equation of motion is derived by from (3.12). It is immediately clear that these Fermions couple to the worldvolume background gauge field strength and do not couple minimally to the gauge field. Since the worldvolume gauge field contains all the gauge fields of the effective action, it follows that the zero mode Fermions of the effective action are uncharged under the $U(1)$ gauge fields of the effective action. 
For our application we require only one left-handed Fermion, that is the neutrino and two other similar particles for the other generations. The neutrino is uncharged under the unbroken $U(1)$ and is therefore compatible with it being a zero mode of (3.12). As the toy model discussed above illustrates the other, charged, particles of the Standard Model should appear in M-theory as solitonic solutions that generalise the embedding solution to include a non-vanishing worldvolume gauge field. The appearance of these states as stretched open strings in the IIA limit ensures the existence of such solutions on the M-fivebrane.

A final problem that we will mention here concerns the masses of the states. As we mentioned states corresponding to matter fields arise a soliton solutions on the M-fivebrane and therefore it is not unreasonable that one could find a spectrum of masses that have a complicated pattern. However, one should also explain the large hierarchy of masses found in the Standard Model which extends over many orders of magnitude even below $M_{W}$. In particular, the electron should appear as just such a soliton, however, one must explain why its mass is six orders of magnitude lower than the mass of the W-bosons. However there is an additional problem in the toy model with the gauge fields. Namely although the toy model has the correct gauge group it has four low energy $U(1)$ vector fields whereas the Standard Model has only one. If the $S U(3)$ factor is made confining, as we discussed above, then this would still leave two $U(1)$ vector fields from the Cartan subalgebra of $S U(2) \times U(1)$. Therefore we need to find some mechanism whereby only a linear combination of these two $U(1)$ vector fields remains massless.

According to the ideas set out in this paper the observable massless fields of the Standard Model, namely the neutrino and the photon, arise as moduli of the M-fivebrane soliton soliton associated with the Fermion and worldvolume gauge field respectively. However, the Fermions of the M-fivebrane are the Goldstone Fermions corresponding to the breaking of the supersymmetry of M-theory by the M-fivebrane. As such the neutrino is a Goldstone Fermion. This is reminiscent of the old suggestion of Volkov and Akulov [41] where the neutrino is a Goldstone Fermion corresponding to the breaking of supersymmetries in four-dimensional spacetime. Although in our case the broken supersymmetries are not those associated to our four-dimensional spacetime. Similarly, here one may regard the photon as a Goldstone boson which is probably related to the breakdown of the central charge symmetries that occur in the eleven-dimensional supersymmetry algebra.

The remaining particles in the Standard Model all carry electric charges and must arise as solitons associated with self-dual strings on the fivebrane which are 
wrapped on the surface $\Sigma$. The charge of these particles arises from the charge carried by the self-dual string. Their mass is given by the rest energy of the soliton and is unlikely to be zero. Indeed, since the massless zero modes of the Mfivebrane are necessarily uncharged with respect to the final low energy $U(1)$ gauge field, there is a link between the electric charge of a particle and its mass. This generalises similar mass inequalities which occur when masses arise in spontaneous symmetry breakdown or when BPS bounds are saturated.

\section{Conclusion}

We have discussed a novel way to derive electro-weak and strong interactions from M-theory and String Theory. In particular we have shown how one can naturally obtain a non-trivial $U(1)$ hypercharge in the low energy effective dynamics of branes. Although we have not presented a concrete model with the correct features of the Standard Model, we have outlined the problems that one faces and also some possible means to over come them. In particular we have shown how the $S U(3) \times S U(2) \times U(1)$ gauge group arises naturally from branes and with matter that has realistic hypercharge assignments. Of course even if a model which satisfies our criteria is found, it would be remarkable if it were to be in agreement with the vast amount of experimental evidence on electro-weak and strong interactions. However, if this is the case then such a model would hold out the very exciting possibility of significant new physics that could test M-theory and String Theory in the next generation of particle accelerator experiments. We also hope that the discussion we have given here will be helpful for other phenomenological applications of branes. We note that the model we described here appears to be significantly harder to use to calculate cross sections and scattering than a standard quantum field theory. Its main advantage is that it is a manifestly finite string theoretic, and so unified, description of Nature that arises in a simple and straightforward manner from M-theory. 


\section{Acknowledgements}

One of the authors (PCW) would like to thank the Universities of Tasmania and Naples for their hospitality where some of this work was carried out and also the Royal Society for a travel grant to Tasmania

\section{REFERENCES}

1. E. Witten, Nucl. Phys. Solutions of Four-Dimensional Field Theories via M-Theory, B500 (1997) 3, hep-th/9703166.

2. P.S. Howe, N.D. Lambert and P.C. West, Classical M-Fivebrane Dynamics and Quantum $N=2$ Yang-Mills, Phys. Lett. B418 (1998) 85, hepth/9710034.

3. N.D. Lambert and P.C. West, Gauge Fields and M-Fivebrane Dynamics, Nucl. Phys. B524 (1998) 141, hep-th/9712040.

4. P. Howe, K. Stelle and P. West, A Class of Finite Four-Dimensional Supersymmertric Field Theories, Phys. Lett. 124B (1983) 55.

5. N. Seiberg, Supersymmetry and Nonperturbative Beta Functions, Phys. Lett. B206 (1988) 75.

6. P. Di Vecchia, R. Musto, F. Nicodemi and R. Pettorino, The Anomaly Term in the N=2 Supersymmetric Gauge Theory, Nucl. Phys. B252 (1985) 635.

7. N. Seiberg and E. Witten, Electomagnetic Duality, Monopole Condensation and Confinement in N=2 Supersymmetric Yang-Mills Theory, Nucl. Phys. B426 (1994) 19, hep-th/9407087.

8. J. Dai, R.G. Leigh and J. Polchinski, New Connections Between String Theories, Mod. Phys. Lett. A4 21 (1989) 2073.

9. J. Polchinski, D-branes and Ramond-Ramond Charges, Phys. Rev. Lett. 75 (1995) 4724, hep-th/9510017.

10. J. de Boer, K. Hori, H. Ooguri and Y. Oz, Kahler Potential and Higher Derivative Terms from M-Theory Fivebrane Nucl.Phys. B518 (1998) 173

11. M. Henningson and P. Yi, Four-Dimensional BPS-specta via M-Theory, Phys.Rev. D57 (1998) 1291, hep-th/9707251

12. A. Mikhailov, BPS States and Minimal Surfaces, Nucl. Phys. B533 (1998) 243, hep-th/9708068. 
13. J.P. Gauntlett, N.D. Lambert and P.C. West, Supersymmetric Fivebrane Solitons, Adv. Theor. Math. Phys. 3 (1998) 1, hep-th/9811024.

14. N.D. Lambert and P.C. West, Monopole Dynamics from the M-fivebrane, hep-th/9811025.

15. I. Antoniadis, N. Arkani-Hamed, S. Dimopoulos and G. Dvali, New Dimensions at a Millimeter to a Fermi ad Superstrings at a TeV, Phys. Lett. B436 (1998) 257, hep-ph/9804398.

16. Z. Kakushadze and S.-H.H. Tye, Brane World, hep-th/9809147.

17. L. Randall and R. Sundrum, Out of this World Supersymmetry Breaking, hep-th/9810155.

18. T. Banks, M. Dine and A.E. Nelson, Constraints on Theories with Large Extra Dimensions, hep-th/9903019.

19. C. Bachas, 'Desert' in Energy or Transverse Space, hep-th/9907023.

20. I. Antoniadis, E. Dudas and A. Sagnotti, Brane Supersymmetry Breaking, hep-th/9908023.

21. L.E. Ibanez and F. Quevedo, Anomalous U(1)'s and Proton Stability in Brane Models, hep-ph/9908305.

22. K. Becker, M. Becker and A. Strominger, Fivebranes, Membranes and NonPerturbative String Theory, Nucl. Phys. B456 (1995) 130, hep-th/9507158.

23. P.S. Howe, N.D. Lambert and P.C. West, The Three-brane Soliton of the M-Fivebrane, Phys. Lett. B419 (1998) 79, hep-th/9710033.

24. H. Bateman, Higher Transcendental Functions, vol. 2, McGraw-Hill, New York, 1953.

25. M. Gogberashvili, Gravitational Trapping for Extra Dimensions, hepph/9808347.

26. L. Randall and R. Sundrum, An Alternative to Compactification, hepth/9906064.

27. N. Arkani-Hamed, S. Dimopoulos, G. Dvali and N. Kaloper Infintely Large New Dimensions, hep-th/9907209.

28. A. Brandhuber and K. Sfetsos Non-Standard Compactifications with Mass gaps and Newtons Law, hep-th/9908116. 
29. P.S. Howe, N.D. Lambert and P.C. West, The Self-Dual String Soliton, Nucl. Phys. B515 (1998) 203, hep-th/9709014.

30. K. Becker, M. Becker, D.R. Morrison, H. Ooguri, Y. Oz and Z. Yin Supersymmetric Cycles in Exceptional Holonomy Manifolds and Calabi-Yau 4-F olds, Nucl. Phys. B480 (1996) 225, hep-th/9608116.

31. G.W. Gibbons and G. Papadopoulos, Calibrations and Intersecting Branes, hep-th/9803163.

32. B.S. Acharya, J.M. Figueroa-O'Farrill and B. Spence, Branes at Angles and Calibrated Geometry, hep-th/9803260.

33. J.L.F. Barbon, Rotated Branes and N=1 Duality, Phys. Lett. B402 (1997) 59, hep-th/9703051.

34. K. Hori, H. Ooguri and Y. Oz, Strong Coupling Dynamics of FourDimensional $N=1$ Gauge Theories from $M$ Theory Fivebrane, Adv. Theor. Math. Phys. 1 (1998) 1, hep-th/9706082.

35. E. Witten, Branes and the Dynamics of QCD, Nucl. Phys. 507 (1998) 658, hep-th/9706109.

36. N.D. Lambert and P.C. West, D-Branes in the Green-Schwarz Formalism, hep-th/9905031.

37. P. S. Howe, E. Sezgin and P. C. West, Covariant Field Equations of the M-Theory Fivebrane, Phys. Lett. B399 (1997) 49, hep-th/9702008.

38. P. S. Howe and E. Sezgin, D=11, $p=5$, Phys. Lett. B394 (1997) 62, hepth/9611008.

39. M.Berger, Sur les Groupes D'holomony des Varieties a Connexion Affine et des Varieties Riemanniennes, Bull. Soc. Math. France 83 (1955) 279; Remarques sur le Group D'holonomie des Varieties Riemannienes, C.R.Sci. Paris 262 (1966) 1316, for a review see S. Salamon, Riemannian Geometry and Holonomy Groups, Longman 1989.

40. J.P. Gauntlett, N.D. Lambert and P.C. West, Branes and Calibrated Geometries, Comm. Math. B202 (1999) 571, hep-th/9803216.

41. D.V. Volkov and V.P. Akulov, Is the Neutrino a Goldstone particle, Phys. Lett. B46 (1973) 109. 